\title{
Crystal structure of (Z)-N-(4-nitrophenyl)-3- phenyl-3-(phenylamino)acrylamide, $\mathrm{C}_{21} \mathrm{H}_{17} \mathrm{~N}_{3} \mathrm{O}_{3}$
}

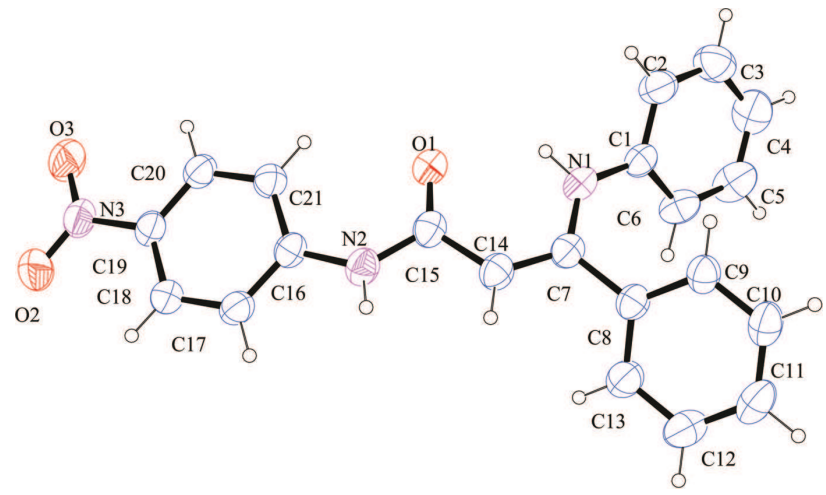

https://doi.org/10.1515/ncrs-2018-0026

Received January 14, 2018; accepted May 3, 2018; available online May 18, 2018

\begin{abstract}
$\mathrm{C}_{21} \mathrm{H}_{17} \mathrm{~N}_{3} \mathrm{O}_{3}$, orthorhombic, Pbca (no. 61), $a=14.3606(4) \AA$, $b=7.4250(2) \AA, c=34.2840$ (9) $\AA, V=3655.62(17) \AA^{3}, Z=8$, $R_{\mathrm{gt}}(F)=0.0399, w R_{\text {ref }}\left(F^{2}\right)=0.1178, T=571(2) \mathrm{K}$.
\end{abstract}

CCDC no.: 1841184

The asymmetric unit of the title crystal structure is shown in the figure. Tables 1 and 2 contain details of the measurement method and a list of the atoms including atomic coordinates and displacement parameters.

\section{Source of materials}

To a screw capped vial with a spinvane triangular-shaped Teflon stir bar were added $N$-(1-phenylethylidene)aniline (0.2 mmol), 4-nitrobenzoyl azide $(0.2 \mathrm{mmol})$ and

\footnotetext{
*Corresponding author: Guo-Kai Jia, Department of Biology and Chemistry, Hunan University of Science and Engineering, Yongzhou Hunan 425199, People's Republic of China; and Hunan Key Laboratory of Comprehensive Utilization of Advantage Plants Resources of Hunan South, Hunan University of Science and Engineering, Yongzhou Hunan 425199, People's Republic of China, e-mail:609158809@qq.com

Xin-Sheng Xiao, Wen-Yi Li and Xiu-Wen Chen: Department of Biology and Chemistry, Hunan University of Science and Engineering, Yongzhou Hunan 425199, People's Republic of China; and Hunan Key Laboratory of Comprehensive Utilization of Advantage Plants Resources of Hunan South, Hunan University of Science and Engineering, Yongzhou Hunan 425199, People's Republic of China
}

Table 1: Data collection and handling.

\begin{tabular}{ll}
\hline Crystal: & Yellow block \\
Size: & $0.25 \times 0.18 \times 0.18 \mathrm{~mm}$ \\
Wavelength: & Cu $K \alpha$ radiation $(1.54178 \AA$ A) \\
$\mu:$ & $7.3 \mathrm{~cm}^{-1}$ \\
Diffractometer, scan mode: & Xcalibur Sapphire 3, $\omega$ scans \\
$2 \theta_{\text {max }}$, completeness: & $125.4^{\circ},>99 \%$ \\
$N\left(h k l_{\text {measured }}, N(h k l)_{\text {unique }}, R_{\text {int }}:\right.$ & $10224,2923,0.022$ \\
Criterion for $I_{\text {obs }}, N(h k l)_{\text {gt }}:$ & $I_{\text {obs }}>2 \sigma\left(I_{\text {obs }}\right), 2426$ \\
$N(\text { param })_{\text {refined }}:$ & 245 \\
Programs: & Bruker programs [1], SHELX [2]
\end{tabular}

1,2-dichloroethane $(1.0 \mathrm{~mL})$ under argon atmosphere conditions. The reaction mixture was stirred at $373 \mathrm{~K}$ for $12 \mathrm{~h}$, filtered through a pad of celite and then washed with ethyl acetate (10 mL 3 times). Organic solvents were removed under reduced pressure and the residue was purified by chromatography on silica gel with ethyl acetate/petroleum (3:1, $v / v)$ as the eluent to give the title compound as a yellow solid.

\section{Experimental details}

All hydrogen atoms were identified in difference Fourier syntheses. The methyl groups were idealized and refined using rigid groups allowed to rotate about the $\mathrm{C}-\mathrm{C}$ bond (AFIX 137 option of the SHELXL program). The $U_{\text {iso }}$ values of all other hydrogen atons were set to $U_{\text {iso }}(\mathrm{H})=1.2 U_{\text {eq }}(\mathrm{C})$.

\section{Discussion}

(Z)- $N$-3-diphenyl-3-(phenylamino)acrylamide derivatives contain attractive structural motifs because of their wide distribution in bioactive molecules and pharmaceuticals [3-6]. Hence, there is considerable interest in the development of effective methods for the synthesis of $(Z)-N$ 3-diphenyl-3-(phenylamino)acrylamide and its analogues $[7,8]$. Herein the crystal structure of the title compound is described to enrich the related crystal structures of (Z)- $\mathrm{N}$-(4-nitrophenyl)-3-phenyl-3-(phenylamino) acrylamide.

Similar to our previous study $[9,10]$, the title compound, built up by the $\mathrm{C}_{21} \mathrm{H}_{17} \mathrm{~N}_{3} \mathrm{O}_{3}$ molecules, has been synthesized. The single crystal structure verifies that all bond lengths are 
Table 2: Fractional atomic coordinates and isotropic or equivalent isotropic displacement parameters $\left(\AA^{2}\right)$.

\begin{tabular}{|c|c|c|c|c|}
\hline Atom & $x$ & $y$ & $z$ & $U_{\text {iso }} * / U_{\text {eq }}$ \\
\hline 01 & $0.65345(9)$ & $0.05732(18)$ & $0.37001(4)$ & $0.0796(4)$ \\
\hline 02 & $0.31779(10)$ & $-0.3752(3)$ & $0.50822(5)$ & $0.1158(6)$ \\
\hline 03 & $0.25421(10)$ & $-0.2581(3)$ & $0.45739(4)$ & $0.1117(6)$ \\
\hline N1 & $0.81351(10)$ & $0.1057(2)$ & $0.32865(4)$ & $0.0739(4)$ \\
\hline $\mathrm{H} 1 \mathrm{~A}$ & 0.7570 & 0.1424 & 0.3311 & 0.089 * \\
\hline N2 & $0.67252(9)$ & $-0.0362(2)$ & $0.43292(4)$ & $0.0706(4)$ \\
\hline $\mathrm{H} 2 \mathrm{~B}$ & 0.7128 & -0.0387 & 0.4515 & $0.085^{\star}$ \\
\hline N3 & $0.32194(11)$ & $-0.2859(3)$ & $0.47814(5)$ & $0.0828(5)$ \\
\hline C1 & $0.84768(11)$ & $0.1020(2)$ & $29017(5)$ & $0.0652(4)$ \\
\hline $\mathrm{C} 2$ & $0.81285(14)$ & $0.2236(3)$ & $0.26350(7)$ & $0.0854(6)$ \\
\hline $\mathrm{H} 2 \mathrm{~A}$ & 0.7682 & 0.3069 & 0.2713 & $0.102^{*}$ \\
\hline C3 & $0.84371(18)$ & $0.2228(4)$ & $0.22515(7)$ & $0.1004(7)$ \\
\hline $\mathrm{H} 3 \mathrm{~A}$ & 0.8194 & 0.3047 & 0.2073 & $120^{*}$ \\
\hline $\mathrm{C} 4$ & $0.90962(18)$ & $0.1019(4)$ & $0.21363(7)$ & $0.1044(8)$ \\
\hline H4A & 0.9316 & 0.1027 & .1881 & $0.125^{\star}$ \\
\hline C5 & $0.94295(16)$ & $-0.0200(4)$ & $0.23976(7)$ & $0.1012(8)$ \\
\hline $\mathrm{H} 5 \mathrm{~A}$ & 0.9876 & 1031 & 318 & 21 * \\
\hline $\mathrm{C} 6$ & $0.91179(13)$ & $-0.0225(3)$ & $0.27762(6)$ & $0.0800(5)$ \\
\hline $\mathrm{H} 6 \mathrm{~A}$ & 0.9342 & -0.1088 & 0.2949 & $0.096^{*}$ \\
\hline $\mathrm{C} 7$ & $0.85638(11)$ & $0.0600(2)$ & $0.36253(5)$ & $0.0638(4)$ \\
\hline $\mathrm{C} 8$ & $0.95999(11)$ & $0.0530(2)$ & $0.36397(5)$ & $0.0641(4)$ \\
\hline C9 & $1.01408(12)$ & $0.1790(3)$ & $0.34450(5)$ & $0.0694(5)$ \\
\hline H9A & 0.9855 & 0.2702 & 0.3303 & $0.083^{*}$ \\
\hline C10 & $1.10992(13)$ & $0.1699(3)$ & $0.34612(6)$ & $0.0841(6)$ \\
\hline $\mathrm{H} 10 \mathrm{~A}$ & 1.1456 & 0.2536 & 0.3326 & $0.101^{*}$ \\
\hline C11 & $1.15295(14)$ & $0.0379(4)$ & $0.36769(7)$ & $0.0942(7)$ \\
\hline $\mathrm{H} 11 \mathrm{~A}$ & 1.2176 & 0.0320 & 0.3687 & $0.113^{*}$ \\
\hline C12 & $1.10006(15)$ & $-0.0853(3)$ & $0.38774(7)$ & $0.0970(7)$ \\
\hline $\mathrm{H} 12 \mathrm{~A}$ & 1.1289 & -0.1736 & 0.4027 & $0.116^{*}$ \\
\hline C13 & $1.00411(13)$ & $-0.0782(3)$ & $0.38563(6)$ & $0.0833(6)$ \\
\hline $\mathrm{H} 13 \mathrm{~A}$ & 0.9688 & -0.1631 & 0.3990 & $0.100^{*}$ \\
\hline C14 & $0.80657(12)$ & $0.0283(2)$ & $0.39545(5)$ & $0.0683(5)$ \\
\hline $\mathrm{H} 14 \mathrm{~A}$ & 0.8394 & 0.0102 & 0.4185 & $0.082^{\star}$ \\
\hline C15 & $0.70617(12)$ & $0.0208(2)$ & $0.39717(5)$ & $0.0661(4)$ \\
\hline C16 & $0.58295(11)$ & $-0.0899(2)$ & $0.44317(5)$ & $0.0609(4)$ \\
\hline C17 & $0.57356(11)$ & $-0.1889(2)$ & $0.47746(5)$ & $0.0684(5)$ \\
\hline $\mathrm{H} 17 \mathrm{~A}$ & 0.6259 & -0.2126 & 0.4926 & $0.082^{\star}$ \\
\hline C18 & $0.48855(12)$ & $-0.2517(2)$ & $0.48914(5)$ & $0.0690(5)$ \\
\hline $\mathrm{H} 18 \mathrm{~A}$ & 0.4827 & -0.3184 & 0.5120 & $0.083^{\star}$ \\
\hline C19 & $0.41173(11)$ & $-0.2144(2)$ & $0.46654(5)$ & $0.0642(4)$ \\
\hline $\mathrm{C} 20$ & $0.41844(11)$ & $-0.1137(3)$ & $0.43327(5)$ & $0.0685(5)$ \\
\hline $\mathrm{H} 20 \mathrm{~A}$ & 0.3653 & -0.0877 & 0.4188 & $0.082^{*}$ \\
\hline $\mathrm{C} 21$ & $0.50398(11)$ & $-0.0507(2)$ & $0.42120(5)$ & $0.0665(4)$ \\
\hline $\mathrm{H} 21 \mathrm{~A}$ & 0.5089 & 0.0174 & 0.3985 & $0.080^{*}$ \\
\hline
\end{tabular}

in normal ranges. The keto-tautomer is found in the solid state (cf. the figure).
Acknowledgements: The work was supported by the HNNSF (No. 2018JJ2143), the opening project of Key Laboratory of Comprehensive Utilization of Advantage Plants Resources in Hunan South, Hunan University of Science and Engineering (Nos. XNZW17C10, XNZW17C05), Research Foundation of Education Bureau of Hunan Province (No. 17A081), Aid program for Science and Technology Innovative Research Team in Higher Educational Institutions of Hunan Province and the NSFC (No. 51772091).

\section{References}

1. Bruker. SMART and SAINT. Bruker AXS Inc., Madison, WI, USA (1998).

2. Sheldrick, G. M.: A short history of SHELX. Acta Crystallogr. A64 (2008) 112-122.

3. Ulrike, S.; Christiane, D.; Claudia, D.; Matthias, F.: Population pharmacokinetics of nintedanib in patients with idiopathic pulmonary fibrosis. Pulm. Pharmacol. Ther. 48 (2018) 136-143.

4. Chauvin, C.; Leruste, A.; Tauziede-Espariat, A.; Andrianteranagna, M.; Surdez, D.; Lescure, A.; Han, Z.-Y.; Anthony, E.; Richer, W.; Baulande, S.; Bohec, M.; Zaidi, S.; Aynaud, M.-M.; Maillot, L.; Masliah-Planchon, J.; Cairo, S.; Roman-Roman, S.; Delattre, O.; Del Nery, E.; Bourdeaut, F.: High-throughput drug screening Identifies pazopanib and clofilium tosylate as promising treatments for malignant rhabdoid tumors. Cell Rep. 21 (2017) 1737-1745.

5. Huang, Z. H.; Li, H. R.; Zhang, Q.; Lu, F. Z.; Hong, M.; Zhang, Z. G.; Guo, X. C.; Zhu, Y. J.; Li, S. M.; Liu, H. Z.: Discovery of indolinone-based multikinase inhibitors as potential therapeutics for idiopathic pulmonary fibrosis. ACS Med. Chem. Lett. 8 (2017) 1142-1147.

6. Tan, L.; Zhang, Z.; Gao, D. L.; Luo, J. F.; Tu, Z. C.; Li, Z. G.; Peng, L. J.; Ren, X. M.; Ding, K.: 4-0xo-1,4-dihydroquinoline3-carboxamide derivatives as new Axl kinase inhibitors. J. Med. Chem. 59 (2016) 6807-6825.

7. He, Z. H.; Liu, W. P.; Li, Z. P.: I $_{2}$-catalyzed indole formation via oxidative cyclization of $\mathrm{N}$-aryl enamines. Chem. Asian J. 6 (2011) 1340-1343.

8. Lei, T.; Liu, W. Q.; Li, J.; Huang, M. Y.; Yang, B.; Meng, Q. Y.; Chen, B.; Tung, C. H.; Wu, L. Z.: Visible light ilnitiated hantzsch synthesis of 2,5-diaryl-substituted pyrroles at ambient conditions. Org. Lett. 18 (2016) 2479-2482.

9. Jia, G. K.; Yuan, L.; Yuan, X. Y.; Li, Z. Y.: Crystal structure of 5-ethyl-2-(p-tolyl)-1,3-dioxane-5-carboxylic acid, $\mathrm{C}_{14} \mathrm{H}_{18} \mathrm{O}_{4}$. Z. Kristallogr. NCS 232 (2017) 819-820.

10. Jia, G. K.; Yuan, L.; Zhang, M.; Yuan, X. Y.; Liu, F.: Crystal structure of 2-(2-bromophenyl)-5-methyl-1,3-dioxane-5carboxylic acid, $\mathrm{C}_{12} \mathrm{H}_{13} \mathrm{BrO}_{4}$. Z. Kristallogr. NCS 231 (2016) 1207-1208. 